\title{
Studies on the Biological Methods of Assaying Streptomycin.
}

\author{
A Comparative Study of Cup-, Paper Disc-, Pulp Disc-, and \\ Superposition-Assay Methods.
}

By

\author{
Nakao Ishida, Ken Katagiri and Reiko Chida. \\ (石田名香雄) (片桐誰) (千田禮子)
}

(Department of Bacteriology, Faculty of Medicine, Tohoku

University. Director: Prof. M. Kuroya.)

(Received for publication, May 29, 1951)

Four agar diffusion methods assaying streptomycin, cup-, paper disc-, pulp disc- and superposition methods, were tested, data obtained were analysed statistically and the results are summarized as follows:

1) It is rather better first to drop the solutions on each of the discs which have previously been put on a large sheet of filter paper, and then to arrange the discs on the agar surface. This fact was confimed statistically with both paper- and pulp disc methods.

2) The pulp disc method is superior to the other two, because, when these methods were compared under the same conditions, the deviations in size of inhibition zones with each plates and the errors due to the whole procedures were found statistically to be least, and the inhibition zones were largest in size in the pulp method.

3) Within an usual standard range of streptomycin units $(1 / 2-16 \mathrm{u} / \mathrm{cc}$.), there exists a relation $y=\mathrm{A} \log x+\mathrm{B}$, where $y$ is the diameter of an inhibition zone produced by streptomycin, $x$ the potency of streptomycin, and A and B constants.

4) When a standard streptomycin solution containing $800 \mathrm{u} / \mathrm{mg}$. was estimated by the pulp disc method using 25 plates, the following excellent results were obtained:

(a) The mean value and its reliable range obtained with each plate was $808.0 \pm 11.2 \mathrm{u} / \mathrm{mg}$; (b) The maximum and minimum probable values of the standard deviation were 29.9 and $15.0 \mathrm{u} / \mathrm{mg}$. (3.76 and $1.83 \%$ ) respectively. When the standard error of the mean obtained from 5 plates was calculated, it was only 1.7 per cent and such an accuracy as obtained by this method was very much higher than those obtained by any other biological test; (c) The analysis of variance showed that a skillful and careful technique especially for diluting standard solutions is essential, to obtain an accurate result.

With regards to the superposition method the probable error was 8 to 10 per cent on an average, while the minimal value being 3.90 per cer.t.

5) Among several plate diffusion methods, we recommend the pulp disc plate method as the best one for streptomycin makers because of its accuracy and simplicity, while the superposition method was useful for experimental and clinical purposes. 